\title{
Study on the Suspension Model and Control of Spherical Driving Joint with Magnetic Levitation
}

\author{
Zeng $\mathrm{Li}^{\mathrm{a},{ }^{*} \text {, Zhang Fan }}{ }^{\mathrm{a}}$, Zhu Zhida ${ }^{\mathrm{a}}$, Zhang Xiao hong ${ }^{\mathrm{a}}$, Chen Fang ${ }^{\mathrm{a}}$ \\ ${ }^{a}$ College of Mechanical Engineering Yangzhou University, Yangzhou 225127, China \\ *Corresponding Author: lizengcf@163.com
}

\begin{abstract}
The traditional machine joints has friction and wear on the mechanical support when they are working, so these make the moving parts hot, and cause the static and dynamic performance worse. Based on the integration of motor, magnetic levitation and robot technology, a new kind of multi degree freedom magnetic levitation active ball joint without mechanical friction, wear and lubrication is presented. The model of electromagnetic levitation force and the electromagnetic torque for the dynamic the joint is established, which is based on the state reconstruction principle and is used to realize the state synchronous suspension control and the self-detection feedback control without sensors. Then the simulation is done, which shows that the system has good dynamic response and anti-disturbance.
\end{abstract}

Keywords: Magnetic levitation, sphere driving joint, the state of reconstruction, synchronous Suspension.

\section{Introduction}

Robots and mechanical arms are usually driven by multiple single-degrees of freedom devices and complex transmission mechanism. They gain three-dimensional motion, which lead to a serious of problems ${ }^{[1,2]}$, such as the increase of complexity, big volume, serious wear of friction surface of each joint, low efficiency, slow response and poor dynamic performance. As an entry point of research, the study of the system of magnetic levitation spherical driving joint can simplify the mechanis $m$, realize the aim of no friction between rotor and stator and no wear of suspending supporting, improve the positional accuracy of joint and response speed and reduce the volume of mechanis $m$ to obtain high response speed. In this research field, magnetic levitation spherical motor and driving joint were first introduced in the world and a number of national patents were applied ${ }^{[3,4]}$.

The research work has a study of mechanism of spherical surface reluctance motor's maglev spherical reluctance driving joint ${ }^{[5]}$, builds magnetic field flux and electromagnetic suspending force and electromagnetic torque model of joint ${ }^{[6,7]}$, and establishes joint ideal spherical rotor three-dimensional dynamics nonlinear coupling model and linearization of decoupling control is performed ${ }^{[8-10]}$. With the limit of rotor pose, instant stepping angle and inability to spin around any axis through center of sphere, its suspension and rotation precision of spherical reluctance driving joint is insufficient. In order to make response speed does not reach the fastest, the final pose of end effectors of joint have to be determined according to the planned track in advance. There-fore, a novel inductive maglev spherical driving joint was presented. Study mechanis $m$ of rotation and suspension and establish a mathematical model of joint system. Analyze the suspension and rotation characters through finite element simulation and testing.

\section{Maglev Spherical Joint Basic Composition}

The 3-D structural drawing of stator and rotor of magnetic levitation spherical driving jo int is shown in Fig.1. Stator 1 and 3 are spherically symmetric and keep coaxial, and they are with functions of driving rotor spin around stator axle and generating maglev force which is able to make rotor along the direction of stator axle. Stator 2 and 4 are spherically symmetric and keep coaxial, and their roles are driving rotor spin around its axle and producing maglev force which can make rotor stable suspending along the direction of rotor axle. Stator 5 was arranged on the bottom of spherical rotor, and its effects are driving spherical rotor spin around stator axle and producing maglev force which 
can make rotor stably suspend along the direction of stator axle. Every stator axle is mutually orthogonal, which produce an intersection that is center of sphere composed by every stator salient pole spherical enveloping surface.

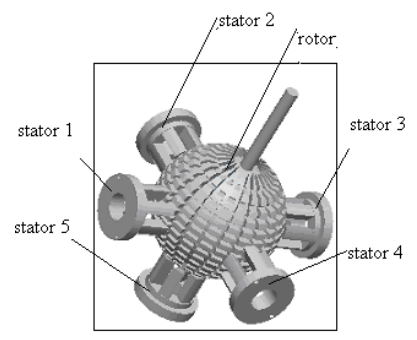

Fig.1 Schematic diagram of maglev spherical driving joint

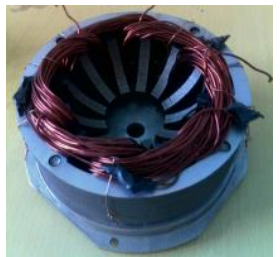

(a) stator

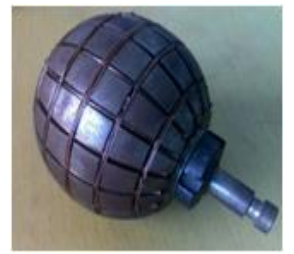

(b) rotor
Fig.2 Stator/Rotor structure

The structural drawing of magnetic levitation spherical driving jo int's rotor and stator is shown in Fig.2. The stator is stacked by $50 \mathrm{~W} 470$ silicon steel sheet. There is a groove, with concentrated distribution winding method, in the middle of stator, embedded with three-phase winding whose connection mode is $\mathrm{Y}$ shape. When there is electricity, each phase winding not only generated electromagnetic torque that can drive rotor revolve but also support radial maglev force for rotor. Spherical rotor, compacted on axle by round nut and shaft shoulder, is stacked also by $50 \mathrm{~W} 470$ silicon steel sheet. On the surface of rotor, there is a mutually orthogonal groove. In the orthogonal point along radial direction to the centre of sphere, there is a hole. The $\mathrm{Cu}$ or $\mathrm{Al}$ is injected into groove and hole and then the armature structure of emanant orthogonal spherical cage shape will be formed in the core of center of sphere short sub and intersect.

\section{Suspension Control Model of Spherical Reluctance Driving Joint with Magnetic Levitation}

From foregoing, the magnetic levitation sphere driving joint is the driving joint which is supported and driven by Multi-Degree-of-Freedom spherical reluctance motor. The research for magnetic levitation sphere driving joint can start at the air-gap magnetic energy between stator and rotor of the motor following the method to study the magnetic reluctance motor. It means that establish the transformational relation of mechanic and electric energy according to the relevance theory of air-gap magnetic energy, and then obtain the relationship between electromagnetic torque of driving rotor and electro magnetic levitation force which can keep the rotor suspend. In order to brief the analysis, as sumes that:

1) three phase stator winding distribute balanced in the space, the magnetic potential generated by each phase current is sinusoidal distribution(or cosine distribution)in air-gap space, and neglect it's higher harmonic component;

2) neglect the effection of short range component group and distribute winding for air-gap magnetic potential, it means that the value of winding factor is 1 ;

3) neglect groove leakage inductance, end leakage inductance and magnetic saturation;

4) neglect the magnetic resistance of iron core and eddy current loss, only the magnetic resistance of operating air-gap is considered in the whole magnetic circuit system.

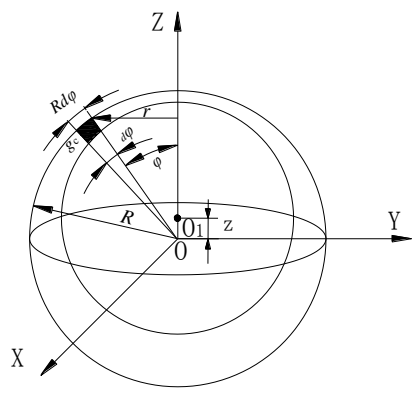

(a)

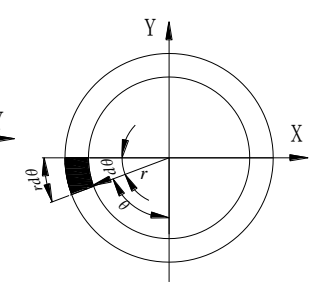

(b)
Fig.3 Schematic diagram of the change of rotor air gap

With the condition that there is no excursion for the center of sphere of rotor of the magnetic levitation sphere driving joint, the radius of spherical surface of stator salient pole is $R$, the radius of spherical surface of rotor salient pole is $R_{r}$, and the gap value between the inner diameter of stator salient pole and the external diameter of rotor salient pole is $g_{0}=R-R_{r}$. Taking the symmetry axis coincides the Z-axial. Establishing the coordinate frame $f(X, Y, Z)=$ $F(R, \varphi, \theta)$. If the center of sphere of rotor moves from point $O$ to point $O_{1}$ under the disturbance of the driving joint, shown in Fig. 3, the excursion along with Z-axial direction is $z$. Taking a micro-spherical-surface in the enveloping spherical surface ring on the six stators, and giving the value of the broad is $R d \varphi$ and the length is $r d \theta$, thus it's aera is $\Delta A=(r d \theta) \cdot(R d \varphi),(\varphi 1<\varphi<\varphi 2,0<\theta<2 \pi)$.

With the rotation of spherical rotor of the magnetic levitation sphere driving joint, the magnetic torque is created by the magnetic pulling force which is generated while one surface of salient pole of rotor overlapped with 
the surface of salient pole of stator partly. Moreover, this magnetic pulling force is the magnetic levitation force which keeps the rotor suspended. Therefore, the variation of air-gap length which be passed by the flux while the flux generated by magnetic field of stator flows to spherical rotor is complexity. And it is a function of $\varphi$ and $\theta$, it is obtained as $\delta_{c}=g_{c}(\phi, \theta)$. As a result, the magnetic conductance is the function of $\varphi$ and $\theta$ which can be defined as $\Lambda(\varphi, \theta)$. According to the method of dealing with the magnetic conductance of the maglev spherical reluctance motor driven by the levitation force in axial plane in document, the unit-area magnetic conductance of the maglev spherical reluctance motor driven by the levitation force in axial plane in this paper is inversely proportional to the gap $e$ which between the stator and the rotor while their magnetic pole of salient pole aligns with each other. Furthermore, it is cosine distribution shown as

$$
\frac{d \Lambda(\varphi, \theta)}{d A}=\frac{K_{0}+K \cos 2(\omega t-\theta-\varphi-\psi)}{e}
$$

where $K_{0}$ and $K$ are magnetic conductance constant which have the relation with the structure of stator and rotor; $\omega$ is the angular speed of the rotor relatives to the stator; $\psi$ is the phase difference between one stator and the rotor while the stator drives the rotor rotating around the coordinate axis of the stator; $e$ is the radial air-gap between the magnetic salient pole of stator and the rotor. Due to the width of the magnetic pole enveloping spherical surface ring on the stator is small, it means the variation range of $\varphi$ $(\varphi 1<\varphi<\varphi 2)$ is small. It is considered that the surface of magnetic pole on stator is parallel with the surface of magnetic pole on the rotor which aligned with the stator, thus e could be shown as

$$
e=g_{0}-z \cos \varphi_{0}
$$

where $\varphi_{0}$ is the angle between the normal line at the position of centroid of stator magnetic poleand $\mathrm{Z}$-axial.

Thus, the magnetic conductance when flux crosses the micro-area $\Delta A$ is

$$
\Delta \Lambda(\varphi, \theta)=\frac{d \Lambda(\varphi, \theta)}{d A} \cdot \Delta A=\frac{K_{0}+K \cos 2(\omega t-\theta-\varphi-\psi)}{e} \cdot \Delta A
$$

Assuming that magnetic potential(magnetic pressure) $h_{s}$ of stator winding generated in air-gap distributes as sinusoidal distribution or cosine distribution ${ }^{[5]}$. It is

$$
h_{s}=H_{s} \cos (\omega t-\theta)
$$

where $H_{s}$ is magnetic potential amplitude, the product of the current $I$ and number of turns of stator $N$, that is $H_{S}=I \cdot N$.

The micro-magnetic-energy generated in air-gap between stator and rotor is

$$
\begin{aligned}
\Delta W_{g} & =\frac{1}{2} \Delta \Lambda(\varphi, \theta) \cdot h_{s}^{2} \\
& =\frac{h_{s}^{2} \cdot\left[K_{0}+K \cos 2(\omega t-\theta-\varphi-\psi)\right] \cdot \Delta A}{2 e}
\end{aligned}
$$

Taking the range of integration $(\varphi 1<\varphi<\varphi 2,0<\theta<2 \pi)$, and getting the integral of above equation, the air-gap total magnetic energy is obtained as

$$
\begin{aligned}
W_{g}= & \int_{0}^{2 \pi} \int_{\varphi_{1}}^{\varphi_{2}} \frac{h_{s}^{2} \cdot\left[K_{0}+K \cos 2(\omega t-\theta-\varphi-\psi)\right] \cdot R r d \theta \cdot d \varphi}{2 e} \\
= & \frac{H_{s}^{2} R^{2} \pi}{2 e} \int_{\varphi_{1}}^{\varphi_{2}}\left[K_{0}+\frac{1}{2} K \cos 2(\varphi+\psi)\right] \sin \varphi d \varphi
\end{aligned}
$$

The equation 6 is the relation of the mechanic and electric energy transformation of magnetic levitation sphere driving joint. It is used to obtain the derivation of the difference of phase between stator and rotor and the displacement in radial direction. Thus, it can obtain the electromagnetic torque and maglev force of magnetic levitation sphere driving joint. The maglev force along Z-axial direction generated by the magnetic levitation sphere driving joint is

$$
F_{z}=\frac{\partial W_{g}}{\partial z}==\frac{N^{2} I^{2} K(\psi) \cdot R^{2} \pi \cos \varphi_{0}}{2\left(g_{0}-z \cos \varphi_{0}\right)^{2}}
$$

where $K(\psi)=-\int_{\varphi_{1}}^{\varphi_{2}}\left[K_{0}+\frac{1}{2} K \cos 2(\varphi+\psi)\right] \sin \varphi d \varphi ; I$ is the current influx in coil; $N$ is the number of turns of stator coil.

Thus it can be seen, the levitation force of magnetic levitation sphere driving joint along $\mathrm{Z}$-axial direction is inversely proportional to the square of the gap $\left(g_{0}-z \cos \varphi_{0}\right)$ which between the salient pole of stator and rotor, and proportional to the square of the magnetic potential Hs generated by coil. Consequently, changing the magnetic potential (or current in the coil) could control the displacement $z$ at $\mathrm{Z}$-axial direction of spherical rotor, and keeping the sphere driving joint rotor suspended stably. As the displacement $z$ of rotor generated, decreasing the current $i$ flows into coils to regulate the position of the rotor and get it returns to the equilibrium position. The current can be shown as $I=I_{0}-i$. When the variation of the displacement is small $\left(z=g_{0}\right)$, linearizing it as

$$
F_{z}=K_{z} \cdot z-K_{i} \cdot i
$$

Where $K_{z}$ is displacement stiffness; $K_{i}$ is current stiffness.

$$
\left\{\begin{array}{l}
K_{z}=\left.\frac{\partial F_{z}}{\partial z}\right|_{\substack{\Delta i=0 \\
z=0}}=\frac{R^{2} N^{2} I_{0}^{2} K(\psi) \pi \cos ^{2} \varphi_{0}}{g_{0}^{3}} \\
K_{i}=\left.\frac{\partial F_{z}}{\partial i}\right|_{\substack{\Delta i=0 \\
z=0}}=\frac{R^{2} N^{2} I_{0} K(\psi) \pi \cos \varphi_{0}}{g_{0}^{2}}
\end{array}\right.
$$


In terms of the Newton's Second Law, the equation of the spherical rotor's movement along Z-axial direction is

$$
F_{z}+F_{d}=m \frac{d^{2} z}{d t^{2}}
$$

Where $m$ is the mass of spherical rotor; $F_{d}$ is disturbance except maglev levitation force.

Substituting equation 8 to 10 , the equation of the spherical rotor's movement along Z-axial direction is obtained as

$$
m \frac{d^{2} z}{d t^{2}}=K_{z} \cdot z-K_{i} \cdot i+F_{d}
$$

According to the law of electromagnetic induction, the relationship of current $i$ and voltage $u$ in winding is obtained as

$$
u=R i+\frac{d \psi}{d i}=R i+L_{0} \frac{d i}{d t}-K_{i} \frac{d z}{d t}
$$

where $L_{0}=\frac{N^{2} R^{2} \pi K(\psi) \cos \varphi_{0}}{g_{0}}$ is electric induction of windings when the rotor at the equilibrium position.

The equation 8 and 12 is the open loop suspension control model of stator sub-system of the magnetic levitation sphere driving joint. The state variable is assumed without disturbance as $x_{1}=z, x_{2}=u, x_{3}=i$.And taking the winding current $i$ as output to get the result that the displacement of rotor is detected without sensor. Thus, according to the equation 11 and 12, the state equation and output equation of open loop is obtained as

$$
\left\{\begin{array}{c}
x \int=A x+b u \\
y=C x
\end{array}\right.
$$

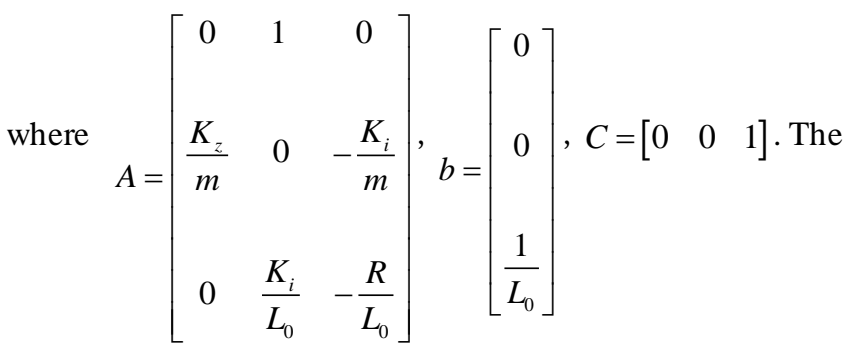

state structure of the systemis shown as Fig.4

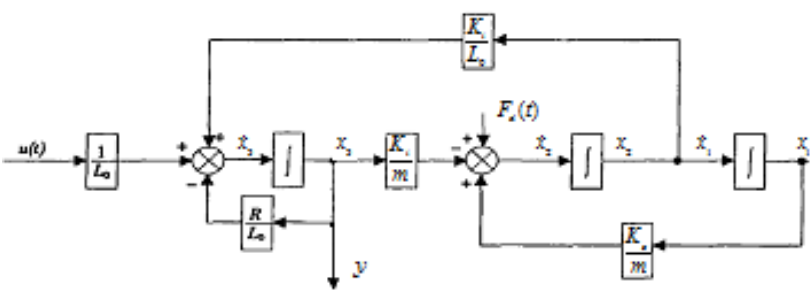

Fig.4 State structure drawing of the stator sub-system

In accordance with the state equation of sub-system (equation 13), the characteristic root of the system is positive real number or positive real part complex number. So, the system is an open loop unstable system which should be took the closed loop integrated correction to keep the suspension system of magnetic levitation sphere driving joint to content the performance requirement.

\section{Design of Synchronous Suspension Control System Based on State Reconstitution}

The structure of synchronous suspension control system with feedback ${ }^{[4,5]}$ with observer constituted by the sub-system (Fig. 4) is shown in Fig. 5. In this drawing, sub-system 1 designs the value of the feedback matrix of output error $G$ based on the principles of state reconstruction, and makes it's state $\dot{x}$ following the state $\grave{x}$ of sub-system 2. Moreover, the follow error of the stable state is zero. The state observer is another sub-system which accomplished by computer or analogue circuit with the selfsame structure with sub-system 1. The purpose of this design is getting the state feedback control of the spherical rotor obtains self-detected displacement at one direction without sensor, as well as realizing the position feedback, speed feedback and the integrated correction control of current feedback.

According to the equation 13, controllability matrix and observability matrix of the system is full rank. It means that the system is completely controllable and observable. Thus, it can be state reconstructed and state feedback controlled.

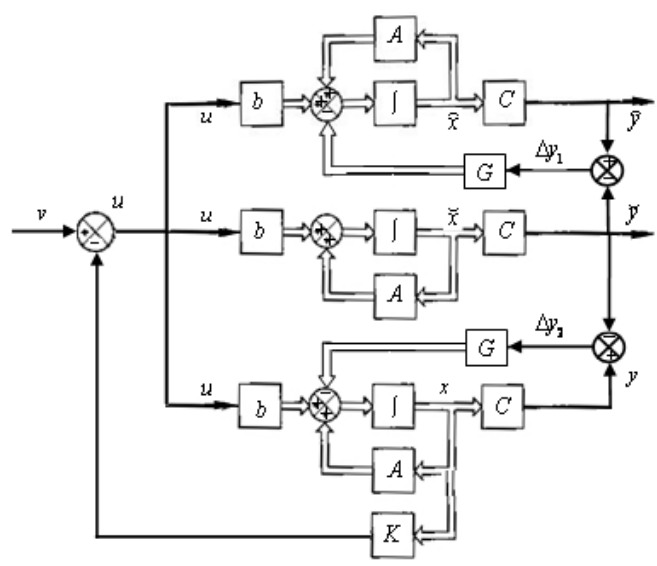

Fig.5 Structure drawing of the synchronous suspension closed loop control based on state reconstruction

In order to driving the spherical rotor to move in synchronism along right about when the two systems come to steady state, and achieving self-detect state feedback control without sensor by the state observer, the output error feedback matrix can be designed according to the principle of state reconstruction to get the two stator sub-systems and the state observer with selfsame state 
variable.

Assuming the output error feedback matrix between the sub-system and state observer is $G=\left[\begin{array}{lll}g_{1} & g_{2} & g_{3}\end{array}\right]^{T}$, the state equation of the two stator sub-systemfrom Fig.5 is

$$
\left\{\begin{array}{c}
\hat{x} \int=(A-G C) \dot{x}+b u+G \grave{y} \\
\grave{x} \int=A \grave{x}+b u
\end{array}\right.
$$

The equation of state observer is

$$
x \int=(A-G C) x+b u+G \grave{y}
$$

Their closed loop characteristic polynomial is

$$
g(\lambda)=\operatorname{det}[\lambda I-(A-G C)]
$$

According to the pole point assigned by the performance requirement of the system, the value of the state synchronous error feedback regulate matrix $\mathrm{G}$ between the two stator sub-systems and the observer can be confirmed by the above equation. Substituting the definite parameters in matrix $A$ and $C$ of the equation (13) to above equation, the characteristic polynomial is obtained as $g(\lambda)=\operatorname{det}[\lambda I-(A-G C)]$

$$
\begin{aligned}
=\lambda^{3}+\left(\frac{R}{L_{0}}+g_{3}\right) & \lambda^{2}+\left(\frac{K_{i}^{2}}{L_{0} m}+\frac{g_{2} K_{i}}{L_{0}}-\frac{K_{z}}{m}\right) \lambda \\
& +\left(\frac{K_{z} K_{i} g_{1}}{m L_{0}}-\frac{R K_{z}}{m L_{0}}-\frac{g_{3} K_{z}}{m}\right)
\end{aligned}
$$

In accordance with the indices of performance of the system, if the pole point assigned at $s_{1,2}=-40$ (key pole point), $s_{3}=400$, the expected closed loop characteristic polynomial of the system is

$$
\begin{aligned}
f(\lambda)= & (\lambda+40)^{2}(\lambda+400) \\
= & \lambda^{3}+480 \lambda^{2}+33600 \lambda+640000
\end{aligned}
$$

Substituting the structure parameter of the system to the equation 17 and 18, and comparing them, the state reconstruction matrix can be obtained as

$$
G=\left[\begin{array}{l}
g_{1} \\
g_{2} \\
g_{3}
\end{array}\right]=\left[\begin{array}{c}
0.482717334 \\
33.6 \\
456.4120348
\end{array}\right]
$$

Assuming the state feedback matrix is $K=\left[\begin{array}{lll}k_{1} & k_{2} & k_{3}\end{array}\right]$, the system's control law is

$$
v=u+K x
$$

After the state reconstruction is achieved, the state variable of each sub-system and obsever and output variable tend to equality when the system comes to steady state. So, the closed loop state equation of the sub-system state feedback is shown as

$$
x \int=(A-b K) x+b y
$$

The closed loop characteristic polynomial is

$$
k(\lambda)=\operatorname{det}[\lambda I-(A-b K)]
$$

Similarly, according to the pole point assigned by the performance requirement of the system, the value of the system's state feedback matrix $K$ can be determined by above equation. Substituting the definite parameter of the matrix $A$ and $b$ in 13 to equation 17 and 18 , characteristic polynomial is obtained as

$$
\begin{aligned}
& k(\lambda)=\operatorname{det}[\lambda I-(A-b K)] \\
& =\lambda^{3}+\left(\frac{R+k_{3}}{L_{0}}\right) \lambda^{2}-\left(\frac{K_{z}}{m}+\frac{k_{2} K_{i}-K_{i}^{2}}{L_{0} m}\right) \lambda-\frac{k_{1} K_{i}+R K_{z}+K_{z} k_{3}}{m L_{0}}
\end{aligned}
$$

Substituting the structure parameter of the system to equation 22 and 18, and comparing them, the state feedback matrix can be obtained as

$$
K=\left[\begin{array}{l}
k_{1} \\
k_{2} \\
k_{3}
\end{array}\right]^{T}=\left[\begin{array}{c}
-102322.801 \\
-30.24 \\
96.7468
\end{array}\right]^{T}
$$

\section{Suspension Control Characters of Spherical Reluctance Driving Joint with Magnetic Levitation}

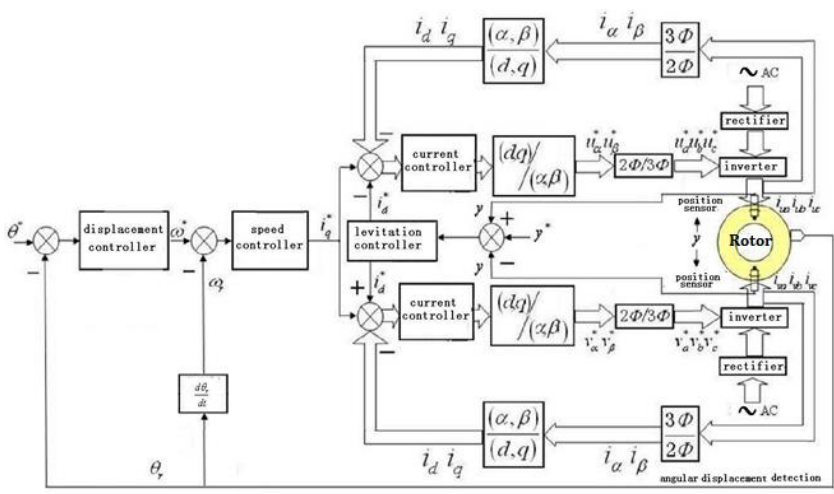

Fig.6 Control system maglev spherical driving join

As shown in Fig 6, design a control-driven system of joint based on the verification of the characteristics of rotation and suspension of inductive maglev spherical driving joint. The system makes a differential drive of spherical rotor through two stators up and down, which makes the rotor to suspend and rotate. The control of rotor's rotating and suspending around $\mathrm{z}$-axes is based on the closed-loop control of motor control system: Input the 
command signal of angular displacement $\theta^{*}$ and the feedback signal of the rotor's angular displacement $\theta_{r}$. Compare the two signals and get the deviation signal, which will be converted to rotational velocity signal $\omega^{*}$ of rotor through angular displacement controller. Comparing $\omega^{*}$ and the feedback signal of the rotor's actual angular velocity, deviation signal of rotational velocity can be got. It will be converted to rotate speed (or torque) to control current $i_{q}^{*}$ by angular velocity controller. At the same time, input the control current signal $i_{d}^{*}$ of suspending force. The signal will be converted to two-phase control voltage by current controller and then be transformed to switch power (inverter) control voltage by a static/active $((d, q) /(\alpha, \beta))$

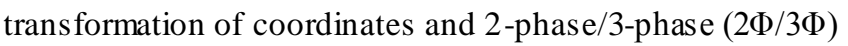
transformation. The voltage will control the input-current of joint windings and adjust the radial displacement (electromagnetic levitation force) and angular displacement (electromagnetic torque) of rotor. Command signal $\theta^{*}$ is used to control rotating angular displacement of rotor while command signal $z^{*}$ is used to control radical displacement

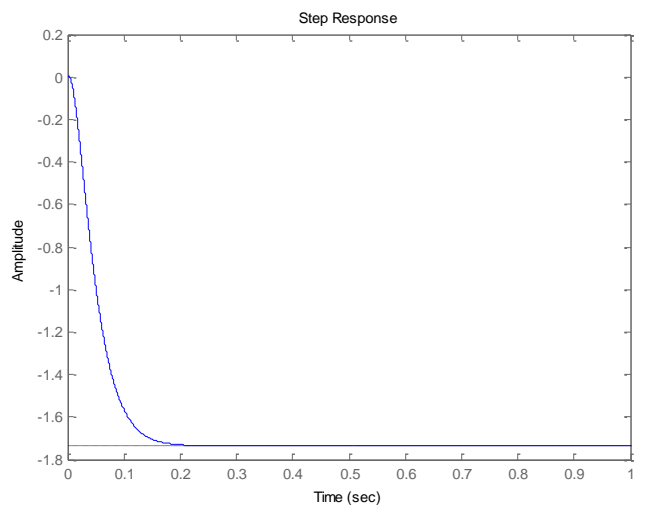

(a)variation of the winding current

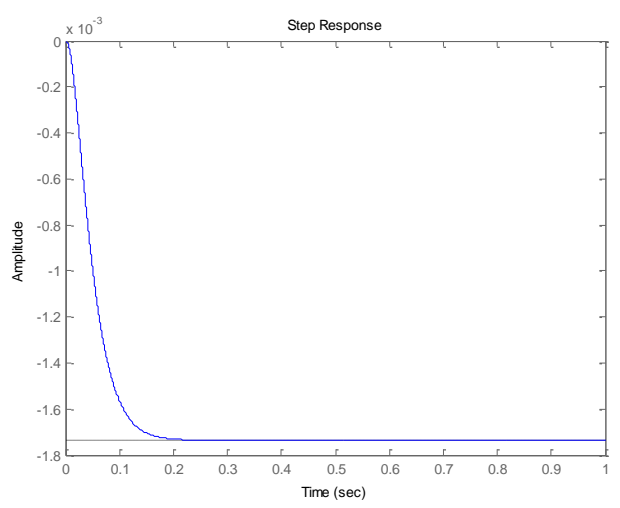

(b)variation of the rotor's displacement

Fig.7 Dynamic response curve of the synchronous suspension of magnetic levitation sphere driving joint

It is the dynamic response curve with step-input signal in Fig.7. It can be seen that, the response speed of the system is rapid. And the adjustment time is less than $0.2 \mathrm{~s}$.
The motion trial of the hot core of spherical rotor deviates from equilibrium position along $\mathrm{Z}$-axial direction while the input signal is sine AC signal with frequency as $50 \mathrm{~Hz}$ in Fig. 8. With the effect of the two stator sub-system's synchronous control, it follows that the deviant of the rotor deviates from equilibrium position is stochastic and quite small which less than $4 \times 10^{-18} \mathrm{~m}$.

After the balance of the rotor, at the time $t=3 \mathrm{~s}$, adding step interference signal at $\mathrm{z}$ direction and observe the displacement at $\mathrm{z}$ direction in Fig. 9. It uses state observer and state feedback control in Fig. 9 (a) and uses PID control in Fig. 9 (b). It can be seen that the two both could get the system back to steady state soon. Using state observer and state feedback control can back to steady state pass by one oscillation. And the biggest deviant is $3.8 \times 10^{-4} \mathrm{~m}$. Using PID control can back to steady state pass by two oscillations. And its biggest deviant is $3.1 \times 10^{-4} \mathrm{~m}$. It is thus clear that basing on the use of state observer and state feedback controller; it has better capacity of resisting disturbance and robustness.

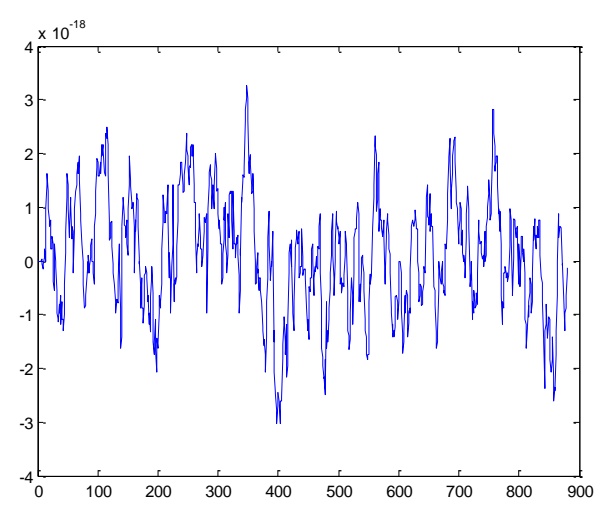

Fig.8 Rator trajectory of departure from balance position on inputting $50 \mathrm{~Hz} \mathrm{AC}$ signal

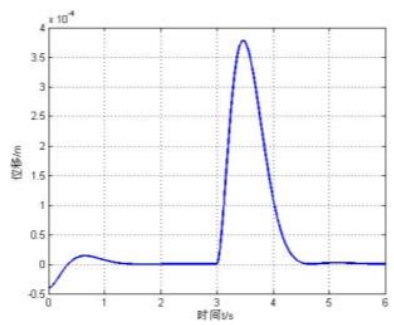

(a)

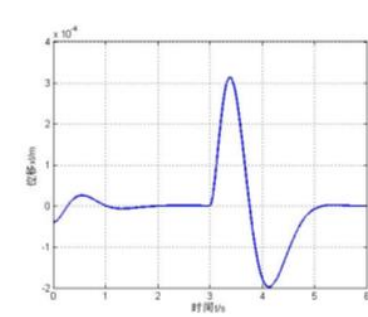

(b)
Fig.9 Interference of step signal in the radial offset

\section{Acknowledgment}

Thank the China Natural Science Foundation for this project. Item Number: 51375427. Thank the Jiang Su Natural Science Foundation for this project. Item Nu mber: 
BK20131232. Thank the Jiang Su Natural Science Foundation for this project. Item Number: BY2014117-08, BY2015061-04.

\section{References}

(1) Mao Xintao, Bao Gang, Yang Qingjun: Joint torque control for the pneumatically robotic manipulator with 3 degrees of freedom, Chinese Journal of Mechanical Engineering, 2008, 44(12).

(2) Li Jian, Li Jianfeng, Wu Zhen: Fault tolerance of redundant manipulators when multi-Joint failed and its optimization, Chinese Journal of Mechanical Engineering, 2002, 38(7).

(3) Zeng Li, Zhang Dan, Dai Min: Maglev spherical re-luctance motor with centripetal thrust or pull, China, 20092003903 2.7. 2001.

(4) Zeng Li, Zhang Dan: Switch reluctance driving joint with magnetic levitation” ,China, 200920039032.7. 2001.

(5) Zeng Li, Wang Jun, Zhang Dan: Mechanism of magnetic force and electromagnetic torque for spherical reluctance motor with magnetic levitation, China Mechanical Engineering, 2011, 2(1).

(6) Zeng Li, Zhang Fan, Zhang Dan: Force and torque study of magnetic levitation spherical driving joint with magnetic field seg-mentation method, Przeglad Elektrotechniczny (Polish) , 2012, 88(7B).

(7) Zhang Fan, Zeng Li, Chen Fang: Study of magnetic levitation spherical joint with decoupling control, Przeglad Elektrotechniczny (Polish), 2012, 88(7B).

(8) Zeng Li, Wang Jun, Xu Yuanyuan: Spherical reluctance driving joint with magnetic invitation modeling and inverse system de-coupling control, Journal of Mechanical Engineering (in Chinese), 2011, 17(9).

(9) Zeng Li, Zhang Fan, Xu Yuanyuan: Three-dimensional dynamical model and controlled characteristics of magnetic levitation spherical reluctance driving Joint, Journal of Mechanical Engineering(in Chinese), 2011, 21(11).

(10) Seiji Shimura, Yohji Okada, Tetsuo Ohishi, Ryou Kondo: Analysis And Control Of An Induction-type levitated rotating motor. Collected Papers of Japanese Society of Mechanical Engineers (C Editor), 61 ( 591 ). 